\title{
A case of hoarseness and vocal cord immobility
}

\author{
Amy M. Trottier MSc MD, Emad Massoud MB, Timothy Brown MD
}

Competing interests: None declared.

This article has been peer reviewed.

Correspondence to:

Timothy Brown,

tfebrown@dal.ca

CMAJ 2013. DOI:10.1503

/cmaj.112112
A 40-year-old woman presented to the otolaryngology clinic with a 7-week history of hoarseness. The symptom had occurred suddenly, and no upper respiratory tract infections or history of vocal overuse immediately preceded the onset. Her voice had not improved during the intervening weeks. She reported symptoms of potential aspiration when drinking thin liquids, and she found herself short of breath when speaking. She had no odynophagia or throat pain, or systemic symptoms such as fever, chills or night sweats. She had not experienced substantial changes in her voice previously.

Three weeks before the onset of hoarseness, the patient had undergone intubation for removal of a benign soft tissue nodule on her left foot. Her voice was normal after the surgery. Her medical history was otherwise unremarkable. She was not taking any medications and had no allergies. She did not smoke and had low alcohol consumption.

On examination, the patient was found to have a breathy, hoarse voice with a self-reported lower pitch than normal. Evaluation of the oral cavity, oropharynx and nasal cavity was unremarkable. Examination of the neck showed no lymphadenopathy. The thyroid examination was normal. Evaluation of cranial nerve function showed no abnormalities other than the hoarseness. Complete left vocal cord immobility was identified on laryngoscopy using a flexible nasopharyngoscope. No laryngeal lesions were found. The patient had a substantial gap between her vocal folds on phona-

\section{KEY POINTS}

- Hoarseness is a common symptom with many different causes, most of which are benign and self-limiting, but some can be serious and lifethreatening.

- In the absence of a firm diagnosis, the best available evidence does not support therapeutic trials of antireflux medications, corticosteroids or antibiotics for hoarseness.

- We suggest referral to an otolaryngologist for visualization of the vocal cords via laryngoscopy within 6 weeks, or as soon as possible if risk factors for malignancy are suspected.

- The left recurrent laryngeal nerve extends into the mediastinum; as such, there are a greater number of worrisome causes of left vocal cord paralysis compared with right vocal cord paralysis.

tion as a result of the immobility (Figure 1).

The cause of the patient's immobile left vocal cord was not readily apparent from her history and physical examination. The 3-week delay between intubation and development of hoarseness made it unlikely that an intubation injury had initially been masked by postoperative laryngeal edema. Computed tomography (CT) from skull base to mediastinum, ordered to rule out malignant lesions along the course of the left vagus nerve and its recurrent laryngeal branch, was normal. Given the negative clinical and imaging work-up, the patient's vocal cord immobility was considered idiopathic.

Because she had substantial symptoms of vocal insufficiency from the glottic air leak, the patient was offered a temporary medialization procedure with injectable hyaluronic acid under local anesthesia by the staff laryngologist. (See Appendix 1, available at www.cmaj.ca/lookup /suppl/doi:10.1503/cmaj.112112/-/DC1 for a diagram of this procedure.) The goal was to improve vocal strength, decrease vocal fatigue and decrease the risk of aspiration while we waited to see if the vocal fold would recover over the subsequent 12 months. The patient underwent the procedure about 8 weeks after symptom onset, with excellent results (Figure 2). Her Voice Handicap Index-10 score ${ }^{1}$ (a self-reported, validated scale) decreased substantially from a score of 31 before the procedure (from a maximum score of 40) to a score of 3 (that of a clinical control) 6 weeks later.

The patient was seen for reassessment at 6 and 12 months after medialization and had at least $90 \%$ recovery of her left vocal cord mobility. This essentially confirmed a neurapraxic injury, because other pathologies would generally not recover spontaneously.

\section{Discussion}

Hoarseness is a common presenting symptom of patients seen by both primary care physicians and otolaryngologists. The term "hoarse" refers to a symptom rather than a specific diagnosis 
and can be used to describe any alteration of the patient's voice from its normal quality. Hoarseness can refer to a rough, raspy, breathy, weak or strained voice, or to changes in pitch and perceived effort.

Hoarseness as a presenting symptom has a broad differential diagnosis, including many causes that are self-limiting or benign, whereas others may be serious and voice-damaging, or even life-threatening. Causes include simple vocal overuse or abuse, irritants such as smoke or chemical exposures, various inflammatory processes, neuromuscular conditions, psychiatric conditions, systemic disorders, neoplasms, or vocal cord lesions or hemorrhage ${ }^{2}$ (Box 1).

The most common cause of hoarseness is acute laryngitis from short-term vocal abuse or an upper respiratory tract infection. Acute laryngitis typically has a rapid onset and is usually self-limiting with a return of normal voice within 1 to 2 weeks. ${ }^{3}$ Our patient had complete left vocal cord immobility, most likely from neurapraxia, a less common diagnosis. We will describe features of unilateral vocal cord immobility later in the discussion.

Information gained from the clinical history can help narrow the differential diagnosis, although direct visualization of the larynx is necessary to make the diagnosis. Key elements of a voice history include whether the hoarseness was gradual in onset or sudden, presence of aggravating or alleviating factors, symptoms of vocal fatigue or throat discomfort when speaking, history of vocal overuse or abuse, antecedent upper respiratory tract infection, recent surgeries requiring intubation and history of similar voice changes in the past. ${ }^{2,4}$ One should ask about symptoms of aspiration, as well as the degree to which the patient uses his or her voice for work, social or sporting activities. Risk factors for head and neck malignancy, such as smoking and heavy alcohol use, or concerning symptoms of dysphagia, odynophagia, hemoptysis or stridor, should be elicited, because these will affect the urgency of further investigation. ${ }^{2,4}$

\section{Investigations for hoarseness}

Visualization of the larynx is an essential investigation for the detection of mass lesions, inflammation or vocal cord mobility issues. ${ }^{5}$ In our patient, laryngoscopy revealed an immobile left vocal cord.

The timing of referral for direct laryngoscopy is controversial. In its clinical practice guideline for hoarseness, the American Academy of Otolaryngology — Head and Neck Surgery Foundation recommends laryngoscopy within a 3month window. ${ }^{5}$ However, critics have noted the

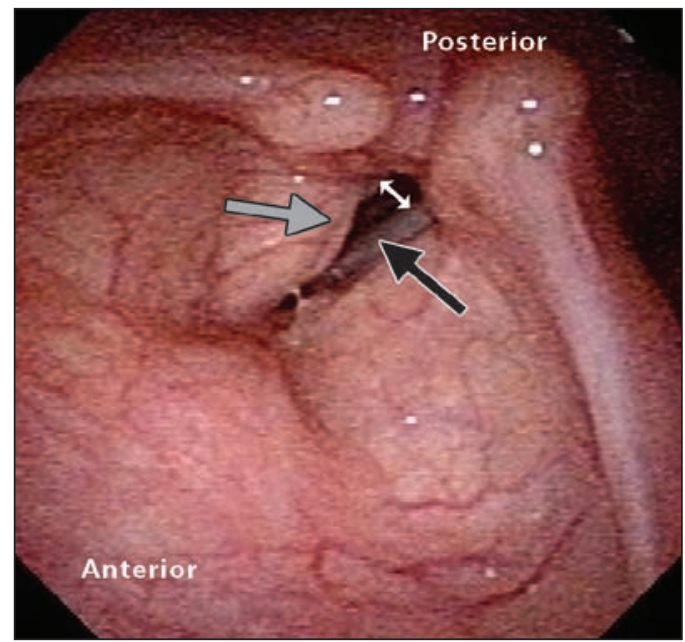

Figure 1: Larynx of a 40-year-old woman with complete left vocal cord immobility (black arrow) showing maximum closure. Note the large glottic gap (white arrow). The grey arrow shows the right vocal cord hidden under the vestibular fold (false vocal cord).
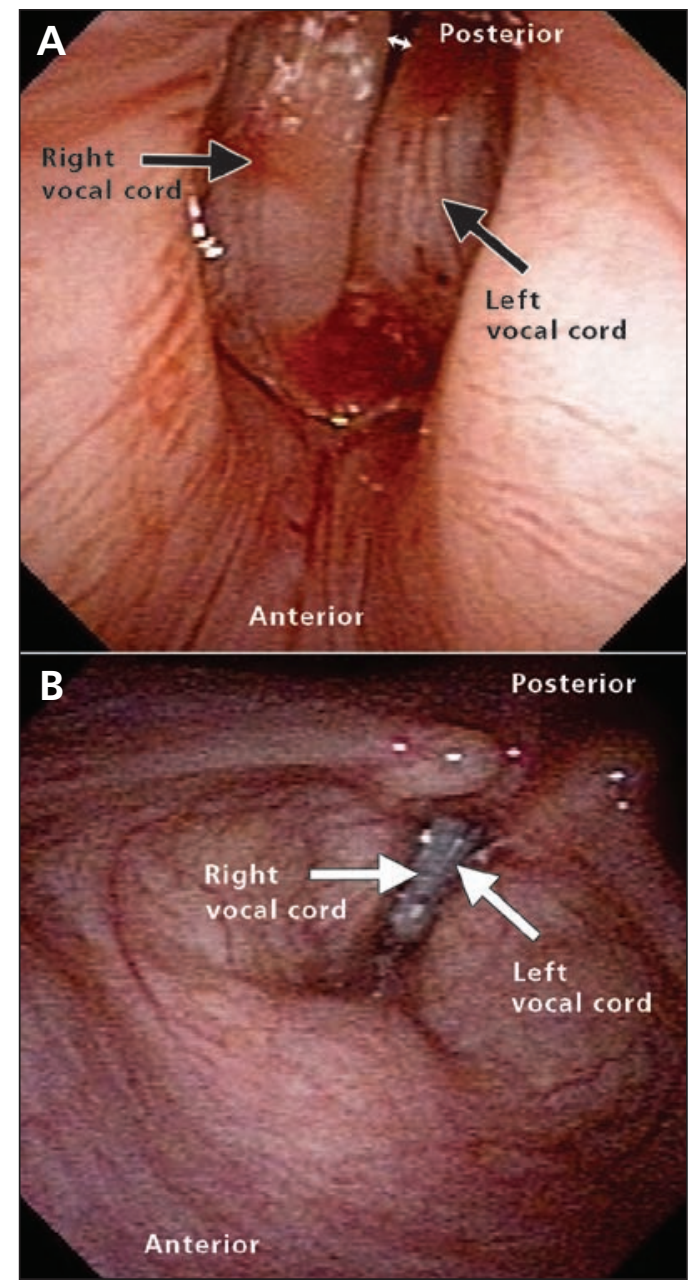

Figure 2: (A) Closure immediately after left vocal cord injection with hyaluronic acid. Other than a minor posterior gap (white arrow), full glottic closure was achieved. (B) Closure 1 month after left vocal cord injection. Full glottic closure is maintained. 
relative lack of evidence (Grade $\mathrm{C}$ ) that supports this recommendation and have expressed concern that the guideline does not emphasize the critical importance of early laryngeal examination in ruling out serious causes. ${ }^{6}$ Based on our experience, we suggest that laryngoscopy be performed within 6 weeks of symptom onset if hoarseness persists. (Please consult your local otolaryngologist as to timing.) During this waiting period, a several-week trial of conservative voice use and vocal hygiene measures is suggested. ${ }^{2,4}$ Conservative voice use refers to the avoidance of shouting, excessive voice use and frequent throat clearing or coughing. Vocal hygiene measures include avoiding irritants such as smoke and dust, reduction in alcohol and caffeine intake, and adequate hydration. ${ }^{2,4} \mathrm{~A}$ patient with a hoarse voice presenting with risk factors for head and neck malignancy or concerning symptoms should be referred more urgently for direct visualization. ${ }^{2,4}$

The guideline recommends against radiologic tests such as CT or magnetic resonance imaging (MRI) before laryngoscopy. ${ }^{5}$

\section{Initial management of hoarseness}

In the absence of concerning risk factors, initial management should include a trial of up to sev-

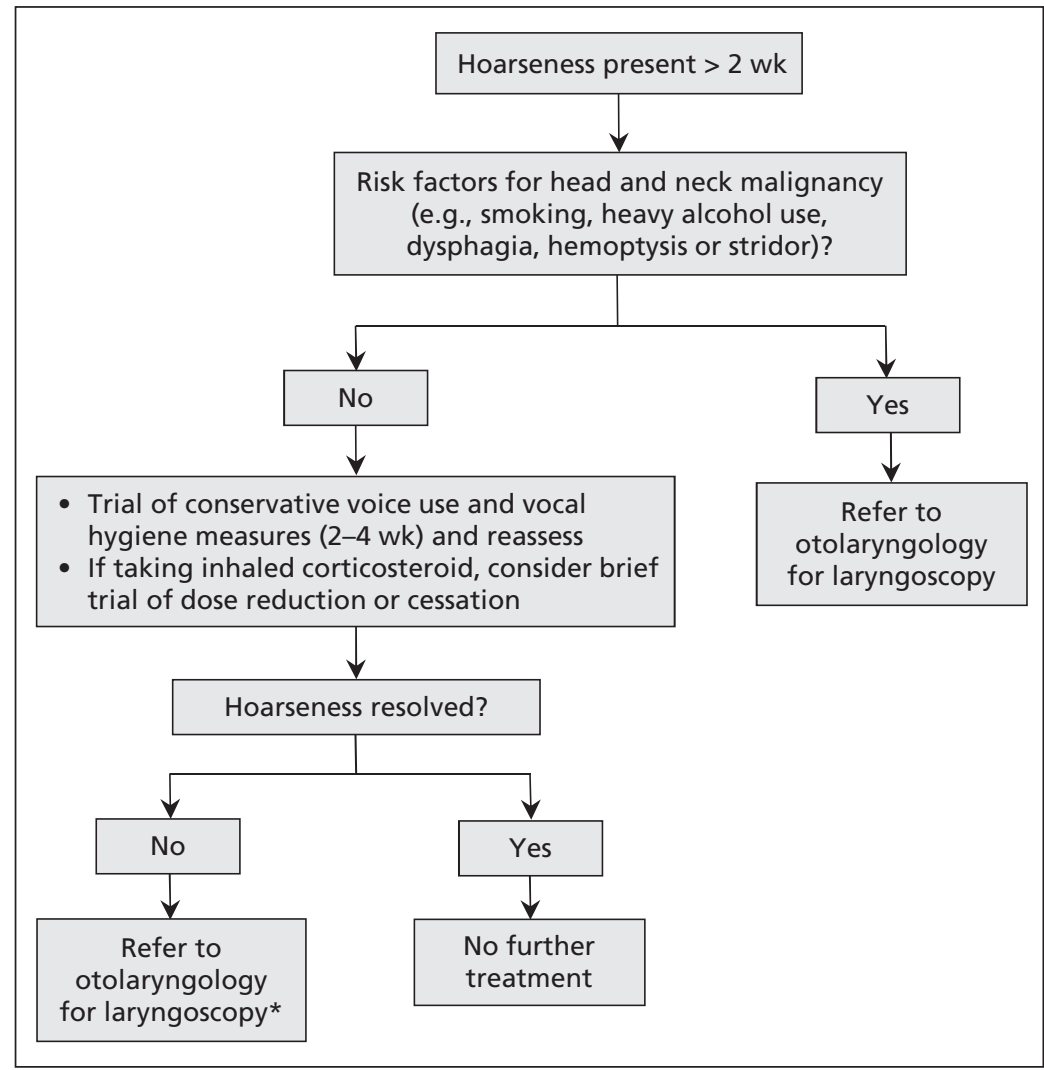

Figure 3: Initial management of patients with a hoarse voice. ${ }^{2,4-8} *$ The ideal timing of laryngoscopy is controversial. (We suggest no more than 6 wk after symptom onset.) Please consult your local otolaryngologist. eral weeks of conservative voice use and vocal hygiene measures before laryngoscopy, as mentioned above. The guideline recommends against therapeutic trials of antireflux medications in the absence of signs or symptoms of gastroesophageal reflux disease, corticosteroids or treatment with antibiotics. 5

For patients using an inhaled corticosteroid, a brief trial of dose reduction or cessation may be considered, because hoarseness occurs with both short- and long-term inhaled corticosteroid use and may be dose-dependent. ${ }^{7.8}$

Based on our experience and after review of the literature, we have developed a simplified flow chart to guide Canadian primary care physicians in the initial management of patients presenting with hoarseness (Figure 3).2.48

Box 1: Differential diagnosis of hoarseness as a presenting symptom

\section{Inflammatory or irritant}

- Allergies

- Upper respiratory tract infections (e.g., viral laryngitis)

- Gastroesophageal/laryngopharyngeal reflux

- Environmental irritants

- Vocal overuse or misuse

- Inhaled corticosteroid

Neoplastic

- Laryngeal papilloma

- Squamous cell carcinoma

- Benign salivary gland malignancies

- Laryngeal dysplasia

- Other laryngeal neoplasms

Neurologic

- Vocal cord paralysis

- Multiple sclerosis

- Myasthenia gravis

- Parkinson disease

- Spasmodic dysphonia

- Essential tremor

- Muscle tension dysphonia

\section{Psychiatric}

- Conversion aphonia

Systemic disorders

- Acromegaly

- Amyloidosis

- Hypothyroidism

- Sarcoidosis

- Rheumatoid arthritis with cricoarytenoid joint involvement

Miscellaneous

- Benign vocal cord lesion (e.g., nodule, polyp, cyst, fibrous mass, hyperkeratosis)

- Vocal cord hemorrhage 


\section{Unilateral vocal cord immobility}

In our patient, we diagnosed complete left vocal cord immobility, most likely from neurapraxia. Symptoms and signs of unilateral vocal cord immobility are due to glottic insufficiency and air escape resulting from a laterally displaced, immobile cord. ${ }^{9}$ This is usually perceived as a breathy, weak voice with variable changes in pitch. The patient's voice may vary from mild hoarseness with vocal fatigue to almost complete aphonia. ${ }^{9}$ Compensation from the opposite vocal cord and false cords can lead to improvement but rarely to complete normalization of the voice.

Aspiration or near aspiration of thin liquids is common. Perceived shortness of breath during conversation and inability to achieve sufficient vocal volume are other common complaints. These symptoms can occur with both vocal cord paralysis and fixation. Vocal cord paralysis indicates a disruption at any point along the path of motor innervation of the larynx, whereas fixation can result from any nonneurologic cause that reduces movement of the cricoarytenoid joint. ${ }^{10}$

\section{Causes}

Any process interrupting the electrical conduction along the recurrent laryngeal nerve or its proximal origin, the vagus nerve, results in paralysis of the ipsilateral vocal cord. The right and left recurrent laryngeal nerves have different courses (Appendix 2, available at www.cmaj.ca /lookup/suppl/doi:10.1503/cmaj.112112/-/DC1). The right recurrent laryngeal nerve loops under the right subclavian artery and then ascends toward the larynx, whereas the left extends into the mediastinum and loops under the ligamentum arteriosum before travelling back to the larynx. ${ }^{10}$ Because the left recurrent laryngeal nerve dips into the mediastinum, there are a greater number of causes of left unilateral vocal cord paralysis. ${ }^{11,12}$ In a retrospective study, the most common cause of unilateral vocal cord paralysis was found to be iatrogenic injury from nonthyroid surgeries (e.g., anterior cervical approaches to the spine and carotid endarterectomies). ${ }^{13}$ Thyroid surgery is the next most common cause. Malignancy was also found to be a substantial cause of left recurrent laryngeal nerve injury. ${ }^{13}$ Intubation can cause injuries to the cricoarytenoid joint and, in rare cases, paralysis of the recurrent laryngeal nerve.

In many cases, a specific cause is not identified and the paralysis is labelled "idiopathic." Many of these cases are believed to be due to postviral neuropathy. ${ }^{11}$

\section{Investigations}

After a unilateral vocal cord immobility is identified on laryngoscopy and in the absence of an obvious cause identified on history and physical examination, additional investigations may be arranged. ${ }^{5}$ The use of imaging studies and their urgency depends on the suspected cause. In cases with a clear temporal relation between symptom onset and preceding trauma, iatrogenic injury or prolonged intubation, imaging is not necessarily warranted. ${ }^{5,11}$ Clinical judgment should always be used to determine the need for imaging on a case-by-case basis.

Imaging must be done from the base of the skull to below the aortic arch so that the full course of both recurrent laryngeal nerves can be visualized. ${ }^{5}$ A CT scan with contrast or MRI are appropriate imaging modalities. ${ }^{5}$ If a more proximal vagal injury is suspected because of findings of palatal paralysis or additional cranial nerve palsies, MRI of the brainstem is warranted. ${ }^{9}$ To differentiate paralysis from fixation, laryngeal electromyography or direct palpation in the operating room are potentially useful investigations.

Thyroid ultrasonography may be a useful additional imaging modality to search for thyroid neoplasms impinging on the recurrent laryngeal nerve. It is generally thought to be a more sensitive technique than CT for the detection of thyroid tumours. ${ }^{14,15}$

\section{Treatment}

The goal of treatment for unilateral vocal cord immobility is to reduce the risk of aspiration and improve vocal quality and strength. Treatment of the underlying cause, if identified, should be undertaken as soon as possible.

Traditional teaching in cases of idiopathic vocal cord paralysis was to wait 1 year before considering intervention to medialize the immobile vocal cord. With the advent of temporary injectable agents such as collagen and hyaluronic acid, temporary medialization of the cord is possible during the waiting period for potential spontaneous recovery. ${ }^{11}$ If no long-term recovery occurs, a permanent surgical implant can be placed in the operating room to medialize the vocal cord and improve glottic closure. ${ }^{11}$

\section{References}

1. Rosen CA, Lee AS, Osborne J, et al. Development and validation of the Voice Handicap Index-10. Laryngoscope 2004;114: 1549-56.

2. Feierabend RH, Malik SN. Hoarseness in adults. Am Fam Physician 2009;80:363-70.

3. Dworkin JP. Laryngitis: types, causes, and treatments. Otolaryngol Clin North Am 2008;41:419-36.

4. Syed I, Daniels E, Bleach NR. Hoarse voice in adults: an evidencebased approach to the 12 minute consultation. Clin Otolaryngol 2009;34:54-8. 


\section{PRACTICE}

5. Schwartz SR, Cohen SM, Dailey SH, et al. Clinical practice guideline: hoarseness (dysphonia). Otolaryngol Head Neck Surg 2009;141:S1-31.

6. Johns MM, Sataloff RT, Merati AL, et al. Shortfalls of the American Academy of OtolaryngologyHead and Neck Surgery's clinical practice guideline: hoarseness (dysphonia). Otolaryngol Head Neck Surg 2010;143:175-7.

7. Yang IA, Fong K, Sim EHA, et al. Inhaled corticosteroids for stable chronic obstructive pulmonary disease. Cochrane DB Syst Rev 2007;(2):CD002991.

8. Adams NP, Bestall JC, Jones P, et al. Fluticasone at different doses for chronic asthma in adults and children. Cochrane DB Syst Rev 2008;(4):CD003534.

9. Sulica L, Blitzer A. Vocal fold paralysis. Berlin: Springer-Verlag; 2006.

10. Lalwani AK. Current diagnosis \& treatment in otolaryngology head \& neck surgery. 2nd ed. New York (NY): McGraw Hill; 2008.

11. Rubin AD, Sataloff RT. Vocal fold paresis and paralysis. Otolaryngol Clin North Am 2007;40:1109-31.

12. Plastiras SC, Pamboucas C, Zafiriou T, et al. Ortner's syndrome: a multifactorial cardiovocal syndrome. Clin Cardiol 2010;33:E99-100.

13. Rosenthal LHS, Benninger MS, Deeb RH. Vocal fold immobility: a longitudinal analysis of etiology over 20 years. Laryngoscope 2007;117:1864-70.

14. Vazquez BJ, Richards ML. Imaging of the thyroid and parathyroid glands. Surg Clin North Am 2011;91:15-32.

15. Wang CP, Chen TC, Lou PJ, et al. Neck ultrasonography for the evaluation of the etiology of adult unilateral vocal fold paralysis. Head Neck 2012;34:643-8.

Affiliations: Division of Otolaryngology — Head \& Neck Surgery (Massoud and Brown), Department of Surgery, Faculty of Medicine, Dalhousie University; and Faculty of Medicine (Trottier), Dalhousie University, Halifax, NS

Contributors: All the authors were involved in the patient's care. Amy Trottier drafted the article, which all the authors revised. All the authors approved the final version submitted for publication.

Acknowledgements: The authors thank Alison Martin for her illustrations, shown in Appendices 1 and 2 .

\section{Change of address}

We require 6 to 8 weeks' notice to ensure uninterrupted service. Please send your current mailing label, new address and the effective date of change to:

\section{CMA Member Service Centre}

1870 Alta Vista Dr., Ottawa ON K1G 6R7

tel $888855-2555$ or $613731-8610 \times 2307$

fax $613236-8864$

cmamsc@cma.ca

\section{Changement d'adresse}

II nous faut de 6 à 8 semaines d'avis afin de vous assurer une livraison ininterrompue. Veuillez faire parvenir votre étiquette d'adresse actuelle, votre nouvelle adresse et la date de la prise d'effet du changement, à l'attention du

\section{Centre des services aux membres de l'AMC} 1870, prom. Alta Vista, Ottawa ON K1G 6R7 tél $888855-2555$ ou $613731-8610$ x2307 fax $613236-8864$ cmamsc@cma.ca

ASSOCIATION
MÉDICALE
CANADIENNE

